www.jmscr.igmpublication.org Impact Factor 5.244

Index Copernicus Value: 83.27 ISSN (e)-2347-176x ISSN (p) 2455-0450 crossref DOI: _https://dx.doi.org/10.18535/jmscr/v4i12.55

\title{
A Hospital Based Study on Serum Uric Acid and Renal Function in Hypertensive Subjects of Northeast India
}

\begin{abstract}
Authors
Dr Nirmali Mattack, Dr Elteza Tahziba Jahir, Dr Tridip Kutum

Dept of Biochemistry \& Dept of Medicine, Gauhati Medical College \& Hospital, Guwahati, Assam

Corresponding Author

Dr Nirmali Mattack

C/O Mr. M.S Khaund, Flat A1, Block 3A, Regent Paradise, Bhetapara, Guwahati-28 INDIA

Email: nirmalimattack@gmail.com, Telephone No.919531004326

Abstract

This is a case control study which involved 50 hypertensive subjects and 50 normotensive subjects and was carried out in Gauhati Medical College and Hospital. Subjects having systolic BP more than 140mm $\mathrm{Hg}$ and Diastolic BP more than $90 \mathrm{~mm} \mathrm{Hg}$ were considered hypertensive. Serum uric acid, urea and creatinine were evaluated in both the case and control groups in VITROS 5600. eGFR was estimated using online calculator based on the UK CKD eGuide on the Renal Association website. Statistical evaluation was done using unpaired student $t$ test and Pearson correlation. The case group had an extremely significant high serum uric acid $(p<0.0001)$ serum urea $(p<0.0001)$ and serum creatinine $(p=0.0009)$ compared to the control group, whereas eGFR was extremely significantly lower in the case group $(p<0.0001)$. A significant negative correlation ( $r=-0.641, p<0.0001)$ was found between serum uric acid and eGFR. This study suggests that uric acid might indeed have a role in mediating hypertension via various mechanisms, and that renal function is closely associated with serum uric acid levels. Therefore, for screening of hypertension, periodic assessment of serum uric acid might be considered.

Keywords: Hypertension, uric acid, urea, creatinine, Egfr.
\end{abstract}

\section{INTRODUCTION}

Hypertension is epidemic in our society and is the most common cardiovascular disease. This increase in hypertension is proportional with the epidemic increase in obesity, metabolic syndrome, type II diabetes, and ESRD, raising the likelihood that these conditions are pathogenetically connected and intricately linked to environmental and especially dietary changes that have occurred in the world population over the past 100 years ${ }^{1}$ Uric acid is a byproduct of purine metabolism produced in blood from endogenous purine $(2 / 3)$ substances or from diet (1/3). Alcoholic and highpurine foods consumption, low water consumption and low physical activity are contributing factors responsible for hyperuriceamia. Its normal level in the body is $<7 \mathrm{mg} / \mathrm{dl}$ in men and $<6 \mathrm{mg} / \mathrm{dl}$ in women, based on the limits of solubility of the monosodium urate in serum at a temperature of $36.80 \mathrm{C}$. The amount of uric acid in the body is affected by the balance of its production and excretion. Conditions associated with uric acid overproduction and reduced renal excretion also causes hyperuricemia. As per Jules et al, urate 
overproduction, which is the primary mechanism for hyperuricemia in 10 percent of the general population, is seen in conditions with high cellular turnover, genetic errors, and tumor lysis syndrome. Inefficient urate excretion, which accounts for 90 percent of cases of hyperuricemia, occurs in renal insufficiency of any cause and with certain medications -. Men have a greater risk of developing hyperuricemia than women in all age groups, although the sex ratio tends to equalize with advancing age. Hyperuricemia is becoming an increasing problem all over the world with a steady increase in its prevalence. ${ }^{2}$

Hyperuricemia has been proposed to have an association with hypertension in various studies. Serum uric acid (UA) levels were demonstrated to be an independent predictor for developing hypertension. Regardless of the different ethnic origins, a continuous relationship between serum UA and blood pressure (BP) was observed in African-Americans and whites as well as in Asians including Koreans. For determining the causal role of serum UA in the development of hypertension, Mazzali et al. demonstrated an elevation in serum UA followed by an increase in $\mathrm{BP}$ via a crystal-independent mechanism in rat models. Reduction of serum UA was associated with a decrease in BP through the regulation of renin-angiotensin and nitric oxide system ${ }^{3}$.

The association of hyperuricemia with hypertension has long been recognized with early investigators such as Frederick Mahomed $^{4}$, Alexander Haig ${ }^{5}$, and Nathan Smith Davis ${ }^{6,7}$, hypothesizing that uric acid might be a cause of hypertension or renal disease. Studies have shown that in overweight and obese subjects, hyperinsulinemia secondary to insulin resistance may enhance the reabsorption of uric acid and thus contribute to the association of hyperuricemia with hypertension ${ }^{8}$. The increasing prevalence of hypertension in Cameroon ${ }^{9-11}$ coupled with the forecast that by the year 2020, non-communicable diseases such as cardiovascular diseases will be the major causes of morbidity and mortality in developing countries, accounting for almost four times as many deaths as from communicable diseases warrant that weight be assigned to the individual risk factors of hypertension and the existence of any possible interaction between them as this will improve the efficiency of prevention strategies ${ }^{2}$.

This study was carried out to investigate any relationship between uric acid and renal function in hypertension in Northeast Indians.

\section{MATERIALS \& METHODS}

This being a case control study consisted of 50 confirmed hypertensive and 50 non hypertensive subjects who came to seek treatment in Gauhati Medical College \& Hospital in the Departments of Biochemistry and Medicine. The study was conducted from January 2016 to August 2016. The inclusion criterion was the diagnosis of essential hypertension on hospital admission. Those with systolic BP of more than $140 \mathrm{~mm} \mathrm{Hg}$ and diastolic BP more than $90 \mathrm{mmHg}$ without the use of anti-hypertensive drugs, or currently taking antihypertensive drugs were taken as hypertensive 12 . Patients were excluded for any of the following reasons: hypertension emergencies requiring intravenous infusion of antihypertensive drugs, severe cardiac insufficiency (grade IV according to the New York Heart Association), acute coronary syndrome, acute cerebrovascular accident, unconsciousness due to any reason, infectious disease, hemodynamic instability due to any reason, receiving diuretics or allopurinol within 2 weeks prior to admission, incomplete data on blood pressure. The study was approved by the Institutional Ethics Committee, Gauhati Medical College \& Hospital and informed written or verbal consent was obtained from all the participants in the study. $5 \mathrm{~mL}$ blood sample was drawn from the participants under aseptic conditions from the median cubital vein. It was collected in properly labelled vacutainers and then centrifuged at $3000 \mathrm{rpm}$ for 15 minutes. The serum thus obtained was subjected to biochemical analysis within 8 hours of collection of blood. Serum uric acid, urea, creatinine were estimated in 
autoanalyser VITROS 5600. eGFR was calculated using online calculator based on abbreviated Modification of Diet in Renal Disease equation ie. $186 \times(\text { Creat / 88.4 })^{-1.154} \times(\text { Age })^{-0.203} \times(0.742$ if female) $\mathrm{x}$ (1.210 if black) from UK CKD eGuide on the Renal Association website. Results were statistically evaluated using student's t test and Pearson's correlation. The results were considered significant when the probability ( $\mathrm{p}$-value) was less than $0.05 \%$ of the observed values of " $t$ " at a particular degree of freedom. Statistical analysis was done using Graph Pad In Stat version 3.00. All the statistical graphs were prepared using Microsoft Excel 2007.

\section{RESULTS}

In our study, the mean value of age in case group is 48.16 years and in control group is 44.64 years which is not significant statistically $(\mathrm{p}=0.110)$.In the case group the frequency was highest $(n==19)$ in the 51-60 years range.

Diagram 1: Frequency of age.

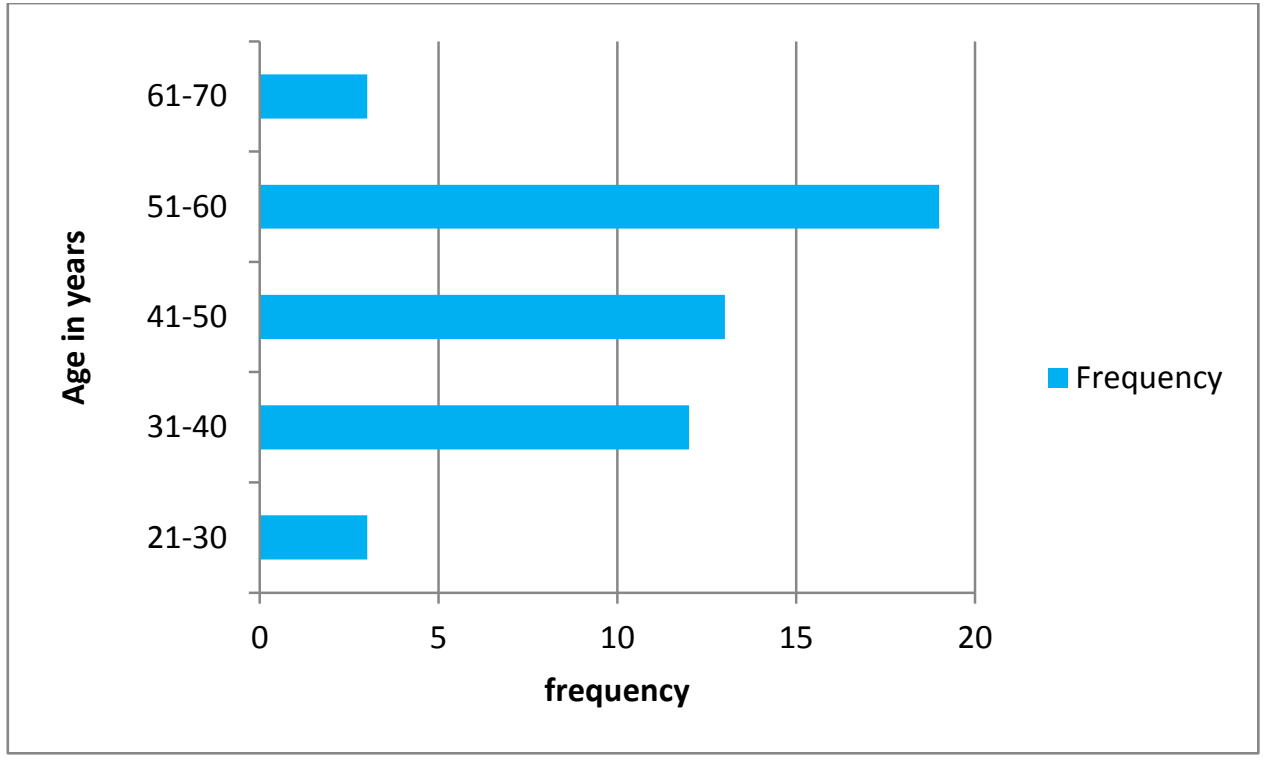

Amongst the 50 cases, $30 \%$ were female subjects while $70 \%$ were male.

Diagram 2: Sex distribution in hypertensives

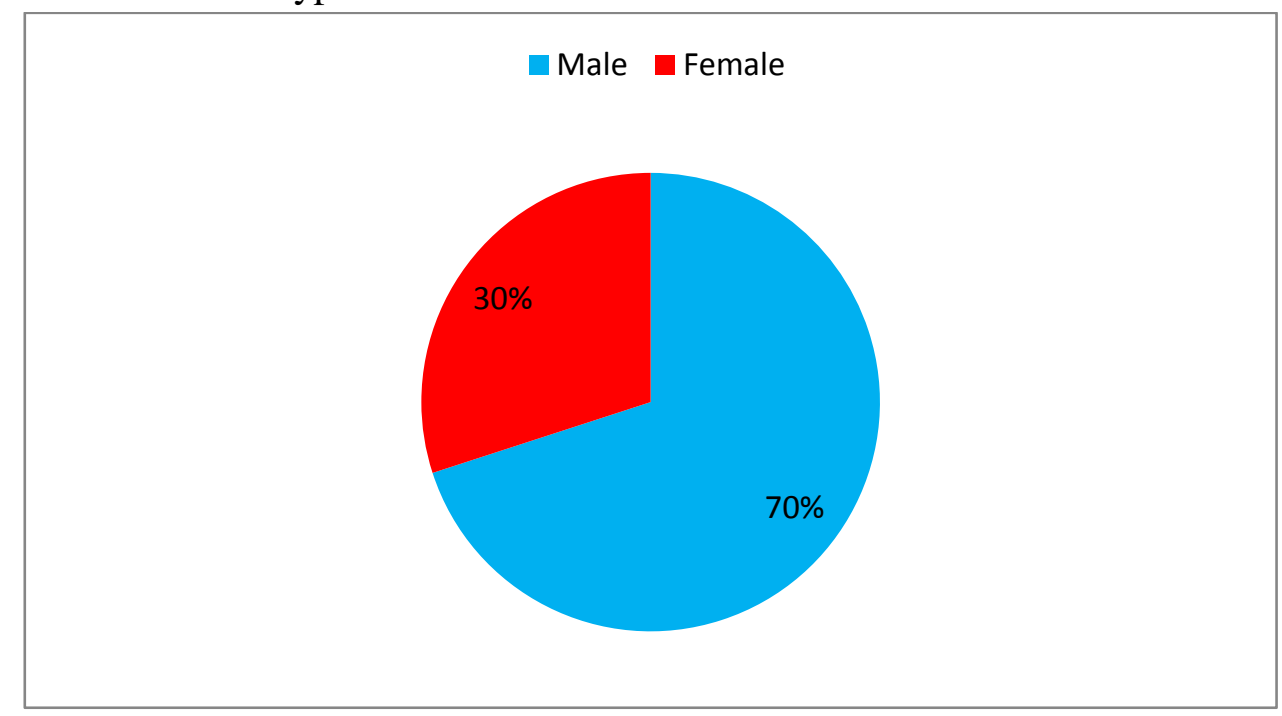

The mean value of creatinine in case was $3.622 \pm$ was considered statistically significant 4.94 while that in control was $1.063 \pm 1.85$ which $(\mathrm{p}=0.0009)$. 


\section{JMSCR Vol||04||Issue||12||Page 14633-14640||December}

Diagram 3: Comparison of means of serum creatinine of case and control groups.

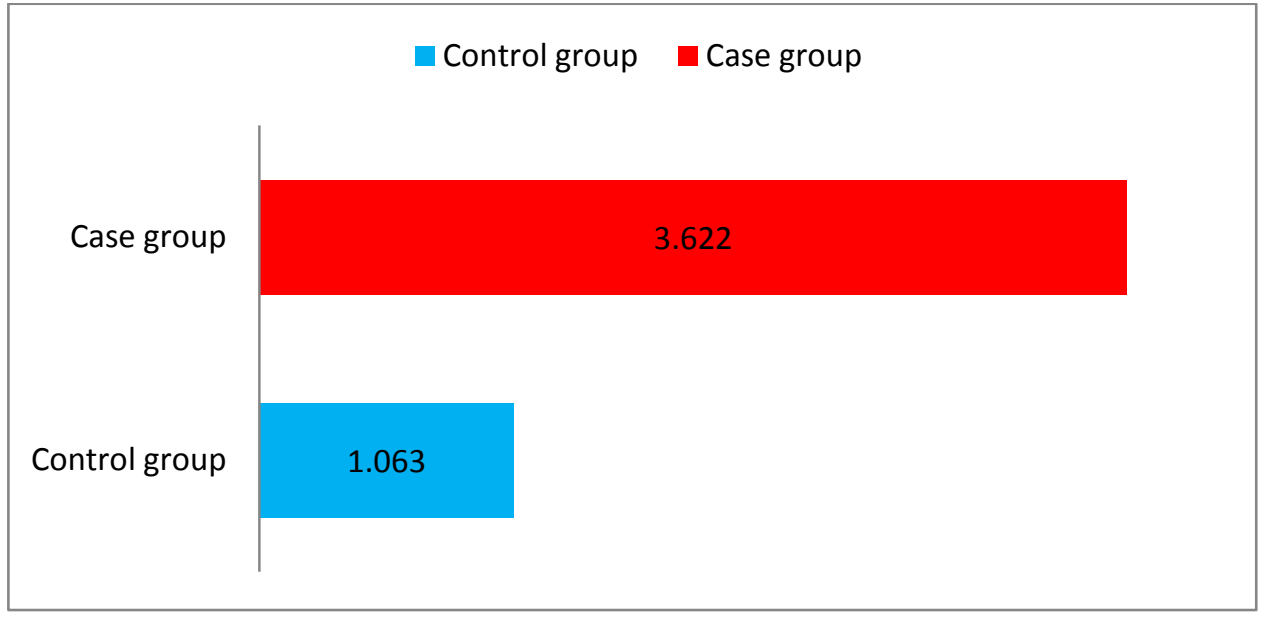

The mean value of urea in case group was $28.562 \pm 26.67$ which was statistically significant $63.915 \pm 55.30$ while that in control group was $(\mathrm{p}<0.0001)$.

Diagram 4: Comparison of means of serum urea of case and control groups

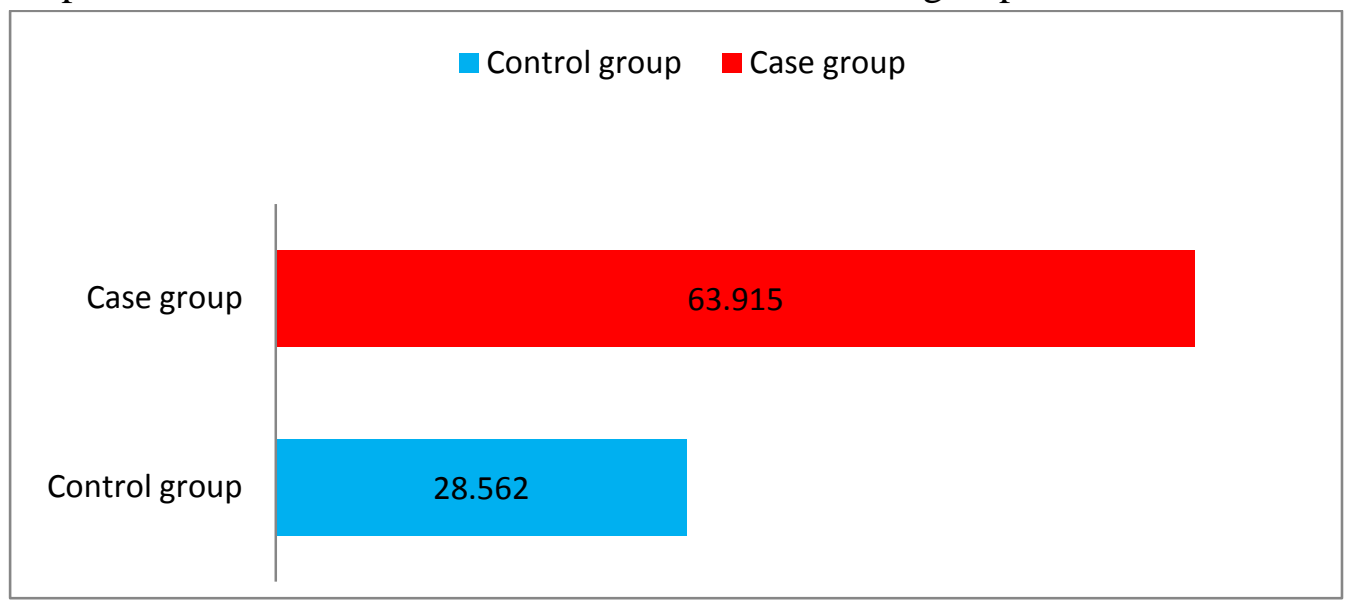

In case of uric acid, the mean value in the case was $4.407 \pm 1.38$ which was also considered group was $6.888 \pm 2.68$ while that in control group significant statistically $(\mathrm{p}<0.0001)$.

Diagram 5: Comparison of means of serum uric acid of case and control groups.

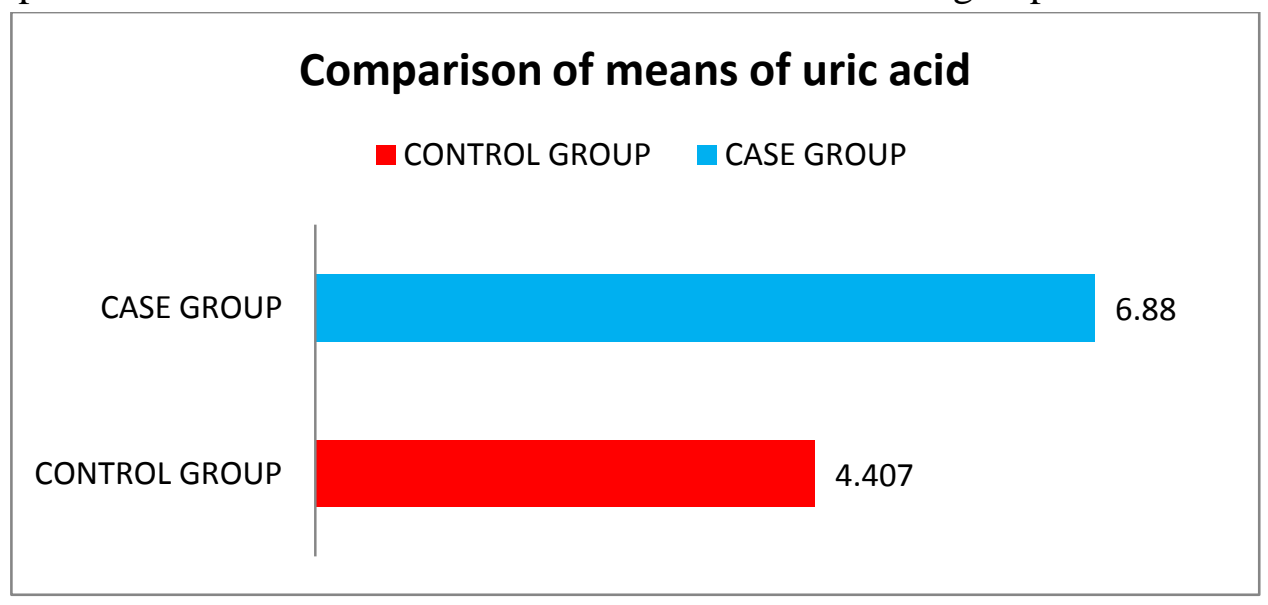

Further we calculated the eGFR in both the case and control groups. The mean value of eGFR in case group was $63.900 \pm 62.04$ while that in control group was $144.00 \pm 88.70$ with a $\mathrm{p}<0.0001$ which was statistically significant. 
Diagram 6: Comparison of means of eGFR of case and control groups.

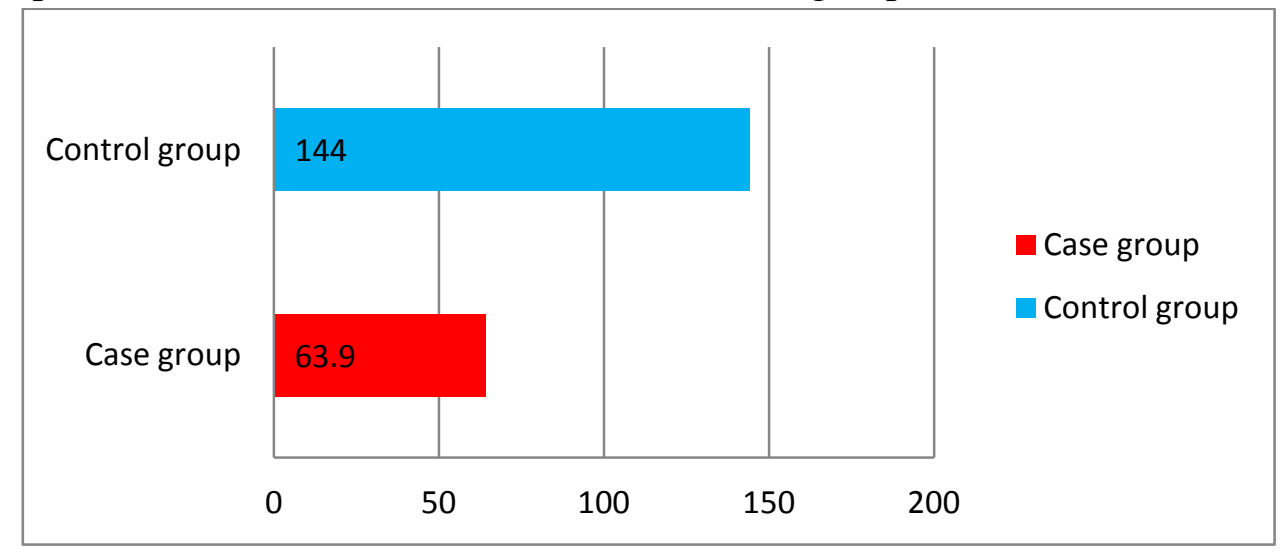

We also recorded the blood pressure in both the pressure in case group was $92.920 \pm 7.61$ while groups. The mean systolic blood pressure in case group was $153.28 \pm 11.07$ while in control group was $122.40 \pm$ 8.43.The mean diastolic blood that in control group was 76.640 \pm 5 .32.In both instances $\mathrm{p}<0.0001$ which was considered statistically significant.

Diagram 7: Comparison of means of blood pressure in the case and control groups

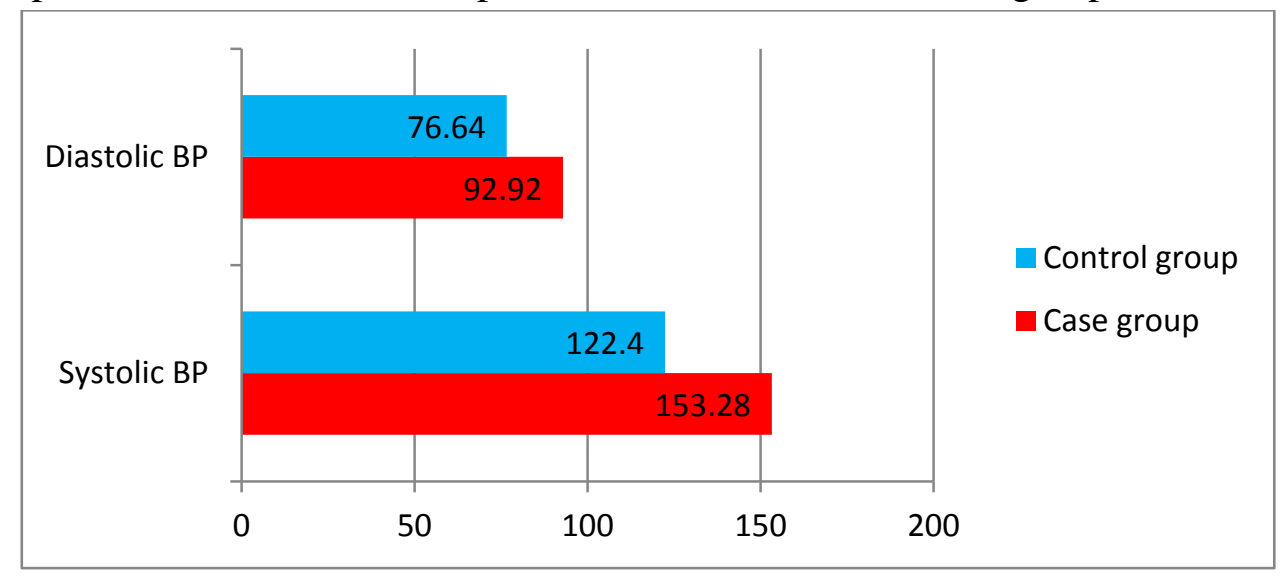

Pearson correlation was done between uric acid and eGFR where we got a positive correlation with $\mathrm{p}<0.0001$ and $\mathrm{r}^{2}=0.4109$.

Diagram 8: Correlation between uric acid and eGFR in the case group.

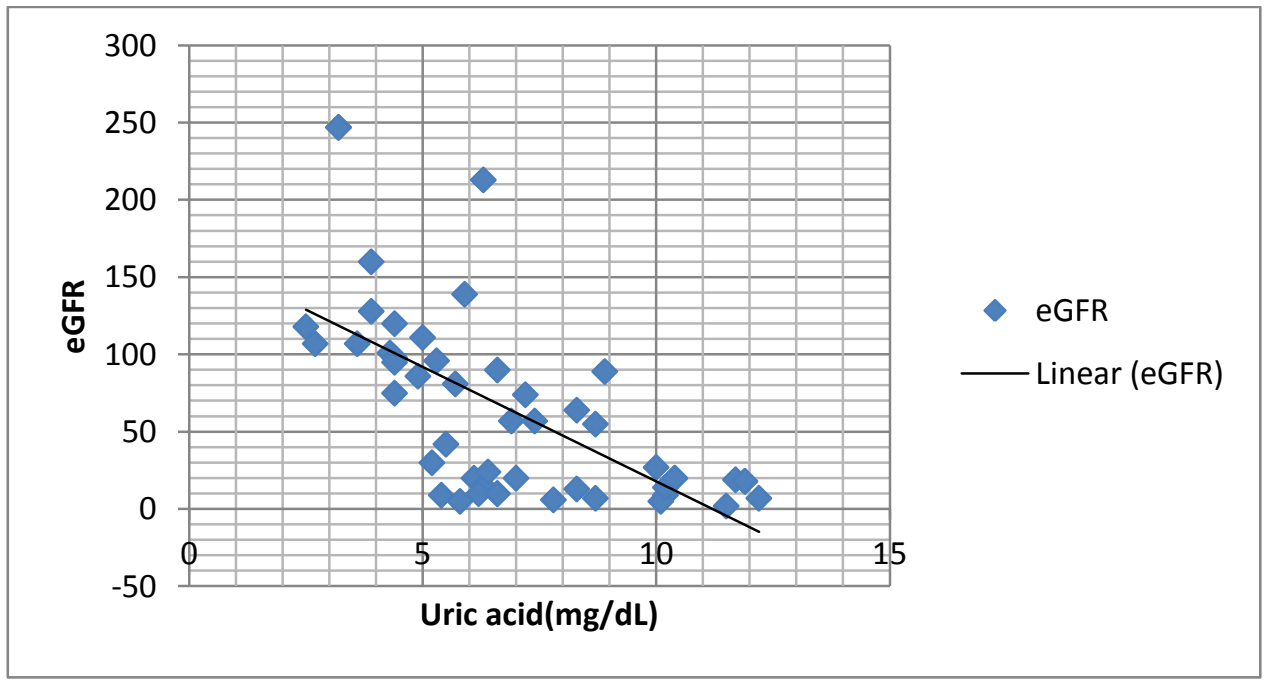




\section{DISCUSSION}

In our study, the mean value of age in case group is 48.16 years and in control group is 44.64 years which is not significant statistically $(\mathrm{p}=0.110)$ thus implying that the two groups are comparable. In the case group the frequency of age was highest $(n==19)$ in the 51-60 years range. This reflects that hypertension is more common in elderly which complies with Logan. With aging, there is a progressive loss of the visco-elastic properties of conduit vessels, increased atherosclerotic arterial disease, and hypertrophy and sclerosis of muscular arteries and arterioles. These vascular changes lead to a loss of the cushioning function of the conduit vessels and stiffening of the arterial vasculature overall, which promote the early return of reflected waves from the peripheral arterial circulation. Early wave reflection amplifies the systolic pressure wave generated with each heart beat, leading to an increase in systolic pressure and a fall in diastolic pressure ${ }^{13}$.

Out of the 50 cases, $70 \%$ were male thereby complying with male dominance in hypertension as per Sandberg. Such a result may be due to women having a more accurate understanding of their health and well-being and thus take the time to seek out health care and will normally follow any treatment that is proposed. However gender differences deserve special attention in the context of hypertension ${ }^{14}$.

Creatinine in case group was significantly higher than in the control group ( $\mathrm{p}=0.0009)$. This finding corroborates with Coresh et al. Elevated serum creatinine level is rare among young and middleaged individuals with optimal blood pressure ( $<1 \%$ prevalence) and becomes increasingly more common among individuals in higher blood pressure categories as well as among individuals using antihypertensive medications. High blood pressure is an important independent predictor of the development and progression of chronic renal disease as well as morbidity and mortality in patients with chronic renal disease ${ }^{15}$.

The mean value of urea in case group was $63.915 \pm 55.30$ while that in control group was
$28.562 \pm 26.67$ which was statistically significant $(\mathrm{p}<0.0001)$. The kidney is both a target and a cause of hypertension. Primary renal disease is the most common etiology of secondary hypertension. Mechanisms of kidney-related hypertension include a diminished capacity to excrete sodium, excessive renin secretion in relation to volume status, and sympathetic nervous system over activity. Conversely, hypertension is a risk factor for renal injury and end-stage renal disease. The increased risk associated with high blood pressure is graded, continuous, and present throughout the distribution of blood pressure above optimal pressure. Renal risk appears to be more closely related to systolic than to diastolic blood pressure $^{16}$.

In case of uric acid also, the mean value in the case group was significantly higher than in the control group $(p<0.0001)$; therefore corroborating with the findings of Neki et al. Although significant epidemiological evidence supported the hypothesis that uric acid may be associated with hypertension, it was not until the experiments of Johnson and colleagues in 2001, established a plausible mechanism. Using a rat model of pharmacologically induced hyperuricemia, they showed that increased serum uric acid level results in hypertension within 2 weeks. The increase in systolic blood pressure (SBP) and diastolic blood pressure (DBP) are proportional to those of uric acid. This can be ameliorated by uric acid lowering drugs (allopurinol or benziodarone). Early hypertension is completely reversible with urate reduction, but prolonged hyperuricemia results in irreversible sodium-sensitive hypertension that becomes uric acid independent. The early hypertension is mediated by increased renal renin activity and reduction of circulating plasma nitrates leading to a phenotype of excessive vasoconstriction that can be reversed by reduction of uric acid or renin- angiotensin system blockade. The later irreversible hyperte-nsion is secondary to altered intrarenal vascular architecture. Uric acid enters vascular smooth muscle cells via uric acid anion transporter-1 
channel, resulting in activation of kinases, nuclear transcription factors, cyclooxygenase- 2 generation, and the platelet derived growth factors (PDGF) and inflammatory proteins (C reactive protein, monocyte chemoattractant protein-1) resulting in the VSCM proliferation, shifted pressure natriuresis, and sodium-sensitive hypertension. If recapitulated in humans, this model suggests that there may be a period of reversible hypertension early in the developmental course $^{17}$.

The mean value of eGFR in case group was $63.900 \pm 62.04$ while that in control group was $144.00 \pm 88.70$ with a $\mathrm{p}<0.0001$ which was statistically significant. Pearson correlation was done between uric acid and eGFR where we got a positive correlation with $\mathrm{p}<0.0001$ and $\mathrm{r}^{2}=0.4109$. This finding corroborates with the findings of Miyatake et al ${ }^{18}$ and $\mathrm{Xu}$ et al ${ }^{19}$.Uric acid level could induce oxidative stress and endothelial dysfunction, resulting in the development of both systemic and glomerular hypertension in association with elevated renal vascular resistance and reduced renal blood flow. In normal rats there was activation of the renin-angiotensin system (RAS), with the development of vascular disease of the afferent arteriolar system (arteriolosclerosis) and glomerular hypertrophy, and over time mild interstitial disease and glomerulosclerosis. Hyperuricemia was also able to induce an epithelial-to-mesenchymal transition, with direct effects on the tubular cell population. As mentioned, the effects of hyperuricemia were particularly impressive in animals with preexisting renal disease, where it accelerated glomerular hypertension and the vascular lesions, resulting in worsening proteinuria and renal failure associated with worsening glomerulosclerosis and tubulointerstitial disease.

Additional studies showed that uric acid might have a role in other experimental models of kidney disease. For example, we found that diabetic mice also developed elevated serum uric acid and that lowering the uric acid level could improve the kidney disease The specific mechanism(s) by which uric acid may be causing these effects has been studied primarily in cell culture systems. One of the more striking findings is that uric acid, while being a potent antioxidant in the extracellular environment, is a pro-oxidant inside the cell where it can induce stimulation of NADPH oxidases with the induction of mitochondrial dysfunction. Uric acid can also induce endothelial dysfunction via a variety of mechanisms and can also stimulate the release of alarmins (such as high mobility group box-1 protein) from endothelial cells that activate Tolllike receptor pathways. Uric acid also stimulates vascular smooth muscle cell proliferation with the production of chemotactic factors and oxidants and the activation of the RAS. Uric acid can also induce phenotypic alterations and chemokines in tubular cells and can induce intrarenal inflammation following its infusion in mice. Further experimental studies outside the scope of this manuscript have found a key role for uric acid not only in CKD, but also in acute kidney injury, metabolic syndrome, non-alcoholic fatty liver disease (NAFLD) and hypertension ${ }^{1}$.

\section{CONCLUSION}

Our study shows a promising negative correlation between uric acid and renal function in hypertensives. Uric acid, by vascular and other mechanisms affect renal function; therefore control of serum uric acid by lifestyle modifications or drugs might be beneficial for both control of blood pressure and for maintainance of renal function. But, drawback of this study is that our findings could be better supported if a follow up study was undertaken and in a larger scale.

\section{REFERENCES}

1. Johnson RJ, Segal MS, Srinivas T, Ejaz A, Mu W, Roncal C, Laura G, Lozada S, Gersch M, Bernardo Rodriguez-Iturbe B, Kang DH, Acosta JH 2005, 'Essential Hypertension, Progressive Renal Disease, and Uric Acid: A Pathogenetic Link?', J Am Soc Nephrol 16: 1909-1919 
2. Nguedia Assob JC, Ngowe MN, Nsagha DS, Njunda AL, Waidim Y, et al. (2014) The Relationship between Uric Acid and Hypertension in Adults in Fako Division, SW Region Cameroon. J Nutr Food Sci 4: 257. doi: 10.4172/2155-9600.1000257

3. Lee JJ, Ahn J, Hwang J, Han SW, Lee KN, Kim JB, Lee S, Na JO, Lim HE, Kim JW, Rha SW, Park CG, Seo HS, Oh DJ and Kim EJ 2015, 'Relationship between uric acid and blood pressure in different age groups'; Clinical Hypertension 21:14

4. Harris ED, Budd RC, Firestein GS, Genovese MC, Sergent JS, et al. (2005) Kelly's Textbook of Rheumatology 7th edn. USA: Elsevier Saunders.

5. Mahomed FA (1979) On chronic Bright's disease, and its essential symptoms. Lancet 1: 399-401.

6. Haig A (1889) On Uric Acid and Arterial Tension. Br Med J 1: 288-291.

7. Davis N (1897) The cardiovascular and renal relations and manifestations of gout. JAMA 29:261-262.

8. Facchini F, Chen YD, Hollenbeck CB, Reaven GM (1991) Relationship between resistance to insulin-mediated glucose uptake, urinary uric acid clearance, and plasma uric acid concentration. JAMA 266: 3008-3011.

9. Mbanya JC, Minkoulou EM, Salah JN, Balkau B (1998) The prevalence of hypertension in rural and urban Cameroon. Int J Epidemiol 27: 181-185.

10. Sobngwi E, Mbanya JC, Unwin NC, Porcher R, Kengne AP, et al. (2004) Exposure over the life course to an urban environment and its relation with obesity, diabetes, and hypertension in rural and urban Cameroon. Int J Epidemiol 33: 769-776.

11. de Ramirez SS, Enquobahrie DA, Nyadzi G, Mjungu D, Magombo F, et al. (2010) Prevalence and correlates of hypertension: a cross-sectional study among rural populations in sub-Saharan Africa. J Hum Hypertens 2: 786-795.

12. Lin F, Zhang H, Huang F, Chen H, Lin C, 3 and Zhu P 2016, 'Influence of changes in serum uric acid levels on renal function in elderly patients with hypertension: a retrospective cohort study with 3.5-year follow-up' BMC Geriatrics 16:35

13. Logan A 2011, 'Hypertension in aging patients', Expert Rev. Cardiovasc. Ther. 9(1), 113-120 (2011)

14. Silva SSBE, Oliveira SFSB, Pierin AMG. The control of hypertension in men and women: a comparative analysis. Rev Esc Enferm USP. 2016;50(1):50-8.

15. Coresh J, Wei GL, McQuillan G, Brancati FL, Levey AS, Jones C, Klag MJ, 'Prevalence of High Blood Pressure and Elevated Serum Creatinine Level in the United States Findings From the Third National Health and Nutrition Examination Survey (1988-1994)', Arch Intern Med. 2001;161(9):1207-1216

16. Yadav R, Bhartiya JP, Verma SK, Nandkeoliar MK, 'Evaluation of blood urea, creatinine and uric acid as markers of kidney functions in hypertensive patients: a prospective study', Indian Journal of Basic and Applied Medical Research; March 2014: Vol.-3, Issue- 2, P.682-689

17. Feig D 2012, 'Advances in Chronic Kidney Disease', Vol 19, No 6 (November): pp 377-385

18. Miyatake N, Shikata K, Makino H, Numata T 2011, 'Decreasing serum uric acid levels might be associated with improving estimated glomerular filtration rate (eGFR) in Japanese men', Vol.3, No.8, 498-503

19. Xu et al 2016, 'The impact of serum uric acid on the natural history of glomerular filtration rate: a retrospective study in the general population'. PeerJ 4:e1859. 